\title{
Range BLU: The Ultimate Companion Robot
}

\author{
A. M. Abdullah Momtaz \\ Major \\ Corps of Electrical and Mechanical \\ Engineers, Bangladesh
}

\author{
Md. Ruhul Islam \\ Senior Manager, \\ Software Development and ERP \\ Support, Brac Bank Limited
}

\author{
A. S. Zaforullah Momtaz \\ Assistant Professor \\ Department of Computer Science \\ \& Engineering \\ University of Asia Pacific
}

\begin{abstract}
This research presented the design and development of an intelligent companion robot called 'Range BLU (Orange Beloved Until) which is well-thought-out as a personal entertainment partner. This robot is equipped with advanced apparatuses and a number of cool sensors for straightforward maneuvers, environment distinguishing and responding accordingly. 'Range BLU uses a number of communication protocols to accept the human expertise and performs a set of unfussy assistance in object handling, appliances executive, and atmosphere control. It is capable to work as entertainment partner in terms of voice, face and object recognition technology via owner's smart phones. In this paper, it has been concentrated on developing 'Range BLU in terms of its operations and functional activities to make a robot more humanoid with the help of human community.
\end{abstract}

\section{General Terms}

Robotics, artificial intelligence, personal assistant, gaming, entertainment.

\section{Keywords}

Robot, portable, Arduino, Bluetooth, speech synthesis, IoT.

\section{INTRODUCTION}

Robotics is going to be the next uprising in the scientific domain. Billions of dollars are being invested by the hi-tech giants for getting the lead in the incredible growth of robotics over the following years. While numerous people are anxious about the potential threats of emotionless machines taking place of humans, the advent of interactive robots has influenced that the machines can be reactive and collaborating as like as humans. Robotics has a long history [1], but the robots with incorporated sensors first appeared in late 70's. With its sensory terminals, these robots were capable to react to their neighboring atmosphere and responded to various scenarios.

After 1999, sensors equipped robots have been renovated to be a new age-bracket machine in the world of robotics. These robots are more intelligent with its advanced computers and sophisticated sensors that help them to interact in a better way and adapt themselves more appropriately to various circumstances. Enrichment in machine learning and invention in computer vision machinery has acquainted with smart functionality that is being used in the interactive robots in this day and age. These robots are more than machines in today's world; they are becoming a smart personal mate daily life.

This research focuses on the development of a brainy robot named 'Range BLU that can offer a myriad of smart functionality through versatile control etiquette for the purpose of personal entertainment and accomplishing micro home tasks. The paper has been demarcated the features and functionality of 'Range BLU and rigorous on the performance analysis based on a number of tests and experiments conducted by the research group.

\section{LITERATURE REVIEW}

Although the discipline of robotics has only been taken to light in the 20th century, the estimate of human invented mechanization has a much prolonged antiquity. In fact, the idea of mechanization by wonder machines had the earliest root in the antique Greek. It is obvious that robotics has practiced a noticeable progression in the 20th and 21st centuries and proficient enhanced functionality and countless promises. Robots have become ubiquitous at the moment from software to hardware. They come in with a myriad of smart functionalities and flavors. Distant from manufacturing or heavy-duty programmed machines, robots are now developed to interact with humans in a more accepted and friendly way through its progressive artificial aptitude.

Robosapien [2] is a major upgrade in the history of recent communicating robotics and was developed as the first intelligent entertainment humanoid. Wow Wee Toys with the special contribution of robot scientist Dr. Mark W. Tilden first presented the science of applied biomorphic automation in a 14-inch recreational robot that can be programmed and mastered with an infrared remote-control communication protocol. Robosapien can perform 67 functions with both movements and sounds. It is responsive to touch and sound signals and can sense obstruction with its interactive impulse system built with six sensors. Robosapien is capable of snatching light objects with its fast and fully articulated arms and facilitate a wide range of motion in movements. Its power saving skill shuts it down when the idle session encompasses more than 120 minutes. Robosapien does not require any setting up - it can be handled with ease and comfort using an ergonomic remote control.

Cozmo [3] is another name in the world of interactive robotics that has been released by Anki, a San-Francisco based company in October 2016. This tiny friend always scans his atmosphere to identify people and recite their names. This robot is exclusive in a sense that it cans illustration intelligent facial countenance. It can be called a sentiment engine for its full range of emotions to respond with human. Cozmo can perceive voice instructions and scan faces to improve one-toone interface. It builds a local $\mathrm{WiFi}$ connection for more secure data transporting, and hence there is a guarantee that Cozmo respects privacy and security. Cozmo can also avoid hindrance through its path and gesture planning. The raw sensor data can be pulling out through SDK if required. 


\section{FUNCTOINAL ACTIVITIES}

This research concentrates to build an all-inclusive collaborative robot called Range BLU that can be functioned with speech command, remote control system and smart phone app, and use artificial intelligence to do some classified jobs itself. To do so, a numerous sensors have been assembled to observe the outer atmosphere to take an appropriate decision on it.

\subsection{Physical Movement}

For a smooth movement as well as navigation, four servo motors [4] have been fitted in the four major junctions like neck, head, and two of the arms right and left side. Also it has a two motors chassis to move from one location to another with the help of four wheels, which helps it to rotate in both the clockwise and counter clockwise alternatively along its own axis.

\subsection{Facial Recognition}

Face recognition [5] is a computer vision application that can detect and identify the face of a particular person from an image or video frame. BLU is equipped with an HD camera and robust face recognition algorithm which allows BLU to act as a security robot for access control system or facility.

BLU captures the image of the face and performs a query in its pre-trained database to find a positive match in the facial features. If the match is positive, BLU gives the approval to get access to a facility or system. BLU has two hands and four wheels, and therefore, it can move smoothly on a plane surface.

With the help of ultrasonic distance sensor, it can detect an obstacle on its path and avoid it with its advanced routing technology. With the help of infrared remote-control system, this robot can be turned on or off along with four basic movement commands like right, left, up, and down.

\subsection{Speech Recognition}

Similarly, BLU can hear and recognize a thousand of voice commands [6]. Therefore, the entire system can be operated through short and precise voice commands. BLU is compatible with Bluetooth based control apps in the Windows, Android as well as Chromium OS platforms.

This app allows the user or the owner to control this robot via Bluetooth from the platforms mentioned above both in the desktop as well as handheld computers. Through this app, BLU can be handled with customizable voice command which provides more flexibility in operation and performance.

\subsection{IoT Operations}

BLU is also capable of connecting with smart home appliances such as air-conditioner, refrigerator, lights etc. and control them with its intelligent communication protocol in terms of IoT [7]. It can also sense fire and give alarms to make everyone aware of the fire breakout.

\section{ALGORITHMS INSIDE 'RANGE BLU}

To advance the functionalities and boost up its maneuvers, the research has settled diverse algorithms, out of those, only three sections on commands engendered by human in the different ways like voice or computer as well as mobile apps, responses in receipt of the sensors and reflection of the input obtained from either the human or sensors have been listed below. All these sections are explained in brief separately as following:

\subsection{Biological Command}

This algorithm fundamentally dictates how BLU receives anthropological command through its three types of communication protocol like vocal command or IoT over internet or App based command over Bluetooth protocol. This machine examines uninterruptedly for a new command. It checks all thinkable command input repeatedly. In the commencement, the robot looks for IoT command, when it becomes false, it examines for a voice command. If none of these command processes is true, then it finds instruction through its serial port. Any true instruction will trigger actions otherwise reject them as the command is found invalid. Figure1 illustrates the flow chart showing the procedure for obtaining a command from its master.

The proposed pseudo-code of the getting human command section is given below:

var key

var inputMethod []$=\{$ IoT, speech, serialPort $\}$

do

inputMethod $=$ getInputMethod ()

if inputMethod $=$ IoT then

takeAction("IoT-INPUT")

else-if inputMethod $=$ speech then

takeAction("SPEECH-INPUT")

else-if inputMethod $=$ serialPort then

takeAction("SERIAL-INPUT")

else

takeAction("DISCARD-INPUT")

end-if

while-end

\subsection{Getting Sensor Reading}

To variety the robot more sensitive to its adjacent atmosphere, it is indispensable to get the sensor readings all at once through an operative algorithm on basis of a consistent runtime routine or schedule. This algorithm has been established for obtaining the data from a set of advanced sensors installed in 'Range BLU includes Camera for visualization. Total 18 sensors are available with the help of smart phones of BLU. The data of all these sensors are stored in respective datasets to be used in real-time or future operations. The proposed pseudo-code of the getting sensor reading section is given below:

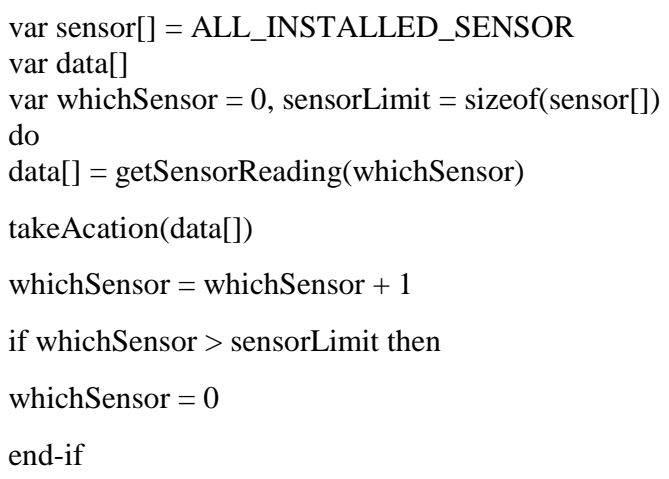


while-end

\subsection{Decision Making}

The most thought-provoking part of a machine is taking pronouncements autonomously according to the given instruction, environment and training dataset. So, the 'Range BLU has been prepared for accomplishing its actions according to the master's instructions and the input obtained from sensors'.

BLU takes actions in 'Take-Action' section and executes accordingly. This component takes all possible orders created by its operator and also the opinion of the contemporary situation around itself with the help of provided sensors. After getting the input, it analyzes and distributes signals to suitable electro-mechanical module or expert unit as essential.

The whole physical body can be classified into three major subsections naming 'chassis' that is proficient of moving the entire body from one position to another, 'arms'- which are used for indicating or holding something as well as to make it interactive too and last one- the 'head' which also has major sensitive components like camera installer in it to make 'Range BLU as a comprehensive machine. Also, this project has a large number of LEDs for representing various status of it in association with audio I/O module naming as 'console'.

The proposed pseudo-code of the decision-making section is given below:

$\operatorname{var} \operatorname{method}[]=\{\operatorname{speech}[]$, sensor[], app[], IoT[] $\}$

var action[]

do

action[] $=\operatorname{get}$ Action $(\operatorname{method}[])$

if action[0] $=$ CHASSIS then

transpose (action[], plane-xy, chassis)

else-if action[0] $=$ ARM then

transpose (action[], plane-z, arm)

else-if action[0] $=$ HEAD then

transpose (action[], plane-xyz, head)

else-if action $=$ CONSOLE then

console (action[audio], action[display])

end-if

while-end

\section{OPERATIONAL DEEDS}

A numeral of action can be accomplished by BLU using human command or sensor readings or the mixture of both of them. 'Path traversing' has been selected as the simplest scenario to exhibit the effective activities of BLU. To move efficiently and accrue the efficacy of the robot, it has to sidestep obstacles over its entire path ways. For that determination, some practical data and scenario has been clarified in this section.

Consequently, the discussion has been divided into two separate subsections including the interaction with human as well as sensor input based solicitation. Both of them are explained below along with adequate table and heuristic figure.

\subsection{Human Interaction:}

In test scenario BLU is started to traverse from node A and the target is to reach at destination node G. The scenario graph is presented in the following figure. In this graph, single circle node represents intermediate node, double circles node represents destination node and rectangle represents the obstacle node. Sequence of traversing is as follows:

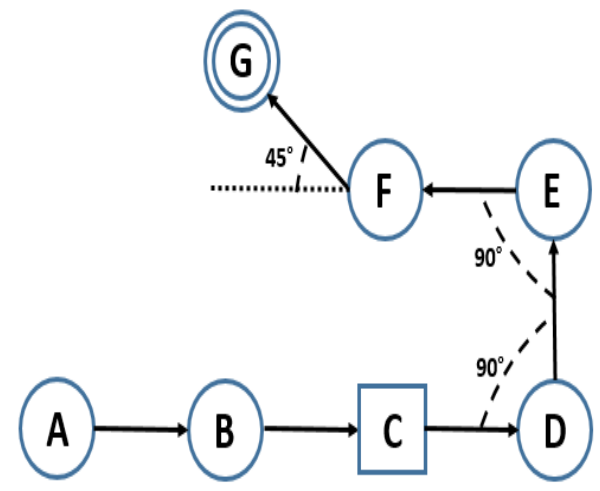

Fig 1: Sequence of traversing by 'Range BLU.

1. Arbitrarily place BLU at A

2. Move forward BLU towards B

3. Move forward BLU D; during motion, scan and measure the shape of the obstacle and try to avoid it at point $\mathrm{C}$

4. At $\mathrm{D}$, rotate $90^{\circ}$ in left in counter clockwise

5. Move forward towards E

6. Rotate again $90^{\circ}$ in left in counter clockwise

7. Move towards F

8. Rotate $45^{\circ}$ in right in clockwise

9. Move forward towards $\mathrm{G}$ (Goal)

10. At G, stop motion

\section{LIMITATIONS \& FUTURE BOOSTS}

Though the projected procedures work fairly well with considerable precision and accuracy, it is allowed that a number of issues confines its functions and disrupts the accuracy of instructions. At first, 'Range BLU does not have legs- it moves with help of wheels.

Therefore, it is incapable of moving appropriately in uneven surface or to another surface level such as stairs or doorsteps. In addition, it is completely based on Google's speech synthesis engine to translate the voice commands into text and vice versa over internet. In many cases, it is being observed that of spelling or accent difference in the voice directions which resulted in a delayed operation.

In the future works regarding this research, it is also intended to improve the voice based knowledge protocol by replacing the Google Speech Recognition with more advanced speech translator to avoid the misleading instructions and make available tolerance for spelling and accent alteration. It is also aimed for enhancing the robot with advanced functionalities by installing different type of hardware or software updates that may be available in future. 


\section{REFERENCES}

\section{[1] Robotics: A}

A Brief History, Https:/Cs.Stanford.Edu/People/Eroberts/Courses/Soco/P rojects/1998-99/Robotics/History.Html

[2] A Fusion Of Technology And Personality, Https://Wowwee.Com/Products/Robots

[3] Big Brain. Bigger Personality, Https://Anki.Com/EnUs/Cozmo.Html

[4] How Servo Motors Work, Https://Howtomechatronics.Com/How-It-Works/HowServo-Motors-Work-How-To-Control-Servos-UsingArduino/

[5] Xiwei Dong, Fei Wu1 And Xiao-Yuan Jing, Generic Training Set Based Multimanifold Discriminant
Learning For Single Sample Face Recognition, Ksii Transactions On Internet And Information Systems Vol. 12, No. 1, January 2018

[6] Nikolaos Flemotomos, Pavlos Papadopoulos, James Gibson, Shrikanth Narayanan, Combined Speaker Clustering And Role Recognition In Conversational Speech, Interspeech 2018, 2-6 September 2018, Hyderabad.

[7] Asst Gokilavani, Ashin Baiju, Unnikrishnan K N, Sunder.V \& Ajay Basil Varghese, A Study On Iot Architecture For Iot Application Domains, International Journal Of Innovative Research In Information Security (Ijiris), Issue 04, Volume 6 (May 2019) 